\title{
INTERVISTA A LUIGI FERRAJOLI
}

La Rivista DESC ha avuto l'onore di colloquiare con il Maestro Luigi Ferrajoli, uno dei filosofi del diritto più autorevoli e più letti nel mondo, con una lunga e notissima storia nella difesa dei diritti fondamentali, sia come professore universitario (Roma, Camerino) sia come giudice negli anni sessanta e settanta, quando partecipò alla fondazione di un'associazione di giovani giudici, "Magistratura Democratica, in difesa delle garanzie fondamentali nel processo, e in particolare nella procedura penale.

È autore di numerossime opere su temi vari, tra i quali diritto e logica, analisi del linguaggio e filosofia della scienza, e è stato il principale discepolo di Norberto Bobbio, fino a consolidarsi come eminente filosofo del "Garantismo", con la diffusione della sua opera di maggiore impatto in Brasile e in tutta l'America Latina, il classico "Diritto e Ragione" del 1989.

Per i suoi meriti e la sua fama è stato insignito del titolo di "doctor honoris causa" in decine di Università in tutto il mondo; la sua monografia più recente è "Manifesto per l'uguaglianza" in 2018, ancora inedita in Brasile.

La possibilità di interpellare il Maestro sui temi di questa intervista è una occasione preziosa, così come ascoltarne il pensiero, e per la Rivista è motivo di orgoglio poter offrire ai propri lettori le riflessioni di Luigi Ferraioli: al Professore dobbiamo non solo la più completa costruzione della teoria del garantismo ma anche la più ricca ed articolata teoria della democrazia costituzionale, e forse nessun paese come il Brasile di oggi ha bisogno del suo insegnamento.

Abbiamo diviso il testo in blocchi tematici in base all'enfasi data dai partecipanti, che il Comitato Editoriale della Rivista DESC ringrazia, per la ricchezza che hanno fornito con le loro domande, cooperando ad un'intervista straordinaria. Questo è ciò che speriamo di offrire ai lettori. 


\section{LUIGI FERRAJOLI - INTERVISTA \\ GARANTISMO E PROCESSO PENALE (parte prima)}

\section{Domanda di EDUARDO JOSÉ DA FONSECA COSTA.}

Giudice federale in Brasile. Dottore presso la Pontificia Università Cattolica di San Paolo (PUC-SP); già presidente dell'Associação Brasileira de Direito Processual (2016-2019) e direttore della Revista Brasileira de Direito Processual.

\section{Domanda 1. Autori come Juan Montero Aroca (Spagna), Franco Cipriani (Italia)} e Alvarado Velloso (Argentina) difendono il processo - civile e penale - come garanzia di libertà al servizio del soggetto giudicato, non come un metodo strumentale al servizio della giurisdizione. Quali sono le somiglianze e le differenze tra il suo ed il loro garantismo?

Luigi Ferrajoli (L.F.): Secondo il modello garantista, il processo giudiziario - civile, penale, ma anche amministrativo e costituzionale - è un procedimento diretto all'accertamento della "verità", ovviamente relativa e approssimativa, in ordine a una violazione del diritto: a un illecito penale, o a un illecito civile o a illeciti di altro tipo, oppure a un atto invalido, sia esso di tipo legislativo o amministrativo o negoziale. Le garanzie processuali sono pertanto - o meglio dovrebbero essere la traduzione giuridica di elementari regole epistemologiche. Sono garanzie di verità, oltre che di immunità dall'arbitrio.

\section{Domande di FLÁVIO BOECHAT ALBERNAZ.}

Membro dell'Ufficio del Pubblico Ministero in Brasile (Stato di São Paulo, procuratore dell'uffizio criminale in São José dos Campos); Master in diritto penale presso la PUC-SP.

Preambolo: In Italia e in Brasile, il Garantismo è stato identificato con una serie di misure di protezione per i criminali, che oppongono severi ostacoli all'applicazione della legge penale e alla punizione efficace e rapida dei reati. Nella pratica forense e nella politica, il suo uso è stato diretto alla tutela del criminale, e non della vittima, o della società, nel caso di reati contro interessi diffusi, ed è stato utilizzato in modo selettivo, dai tribunali dal Parlamento, soprattutto per criminali speciali, come i detentori del potere politico ed economico. Ad esempio, in Italia, nel luglio del 1994 nel contesto delle indagini condotte da parte del pool di pubblici ministeri di "Mani Pulite", il governo presentò un decreto legge per cambiare in modo sostanziale le norme sulla detenzione cautelare quanto ai reati finanziari, di corruzione e altri reati dei quali erano state accusate proprio le persone coinvolte nello scandalo noto come Tangentopoli. In pratica, chi avesse rubato poche cose in una farmacia era suscettibile di essere soggetto alla custodia cautelare, chi avesse malversato miliardi (la moneta corrente in Italia all'epoca era la lira) dalle casse dello Stato o da altre società avrebbe invece potuto avere garantita la libertà. In Brasile, in particolare nei tribunali superiori, alcuni giudici hanno agito sotto forte conflitto di interessi (ci sono giudici dei tribunali superiori legati ad "imputati eccellenti" da legami politici e di amicizia), e il garantismo è stato invocato selettivamente, come pretesto per giustificare il 
divieto del carcere cautelare per accusati o indagati speciali, ovvero per garantire l'allungamento dei processi in vista della prescrizione e dell'impunità.

Fatta questa breve introduzione, chiediamo:

\section{Domanda 2. Questa è una percezione corretta? Se non lo è, dov'è sbagliata?}

L.F.: Si tratta di una percezione del tutto sbagliata: le garanzie penali e processuali sono a tutela degli innocenti e non già dei criminali. E' perciò infondato qualunque appello al garantismo a sostegno dell'impunità dei reati dei potenti. "Garanzie" e "garantismo", d'altro canto, sono espressioni il cui significato va ben al di là del diritto penale. Le garanzie sono gli obblighi o i divieti corrispondenti a quelle aspettative positive o negative che sono i diritti soggettivi. In particolare, sono le tecniche di tutela di tutti i diritti fondamentali, cioè i divieti (o limiti) e gli obblighi (o vincoli) imposti quali loro garanzie. Parleremo perciò di garantismo liberale e specificamente penale per designare i limiti all'arbitrio punitivo, sia legislativo che giudiziario o poliziesco, a garanzia dei diritti di libertà; di garantismo sociale per designare gli obblighi di prestazione corrispondenti ai diritti sociali; di garantismo civile per designare i limiti e i vincoli imposti ai poteri economici privati - in materia di lavoro, di ambiente, di sicurezza e simili a garanzia dei diritti di quanto possono essere lesi dal loro esercizio sregolato; di garantismo sovranazionale e tendenzialmente globale per designare le garanzie, quasi totalmente mancanti, correlative ai diritti fondamentali stabiliti dalle tante carte e convenzioni internazionali e finora sostanzialmente inefettive; di garantismo dei beni pubblici o fondamentali contro la loro lesione a danno dei diritti di tutti.

In questo senso il garantismo è l'altra faccia-la faccia dell'effettività-del costituzionalismo. E' un insieme di limiti e vincoli imposti a qualunque potere, sia esso pubblico o privato, politico o giudiziario, statale o sovrastatale, a garanzia delle diverse classi di diritti soggettivi e, in particolare, dei diritti fondamentali: dei diritti politici, dei diritti civili, dei diritti di libertà e dei diritti sociali. Non è quindi un ostacolo all'applicazione della legge penale, ma al contrario una garanzia della sua correttezza e perfino della sua effettività quale strumento di tutela delle vittime e della società. L'effettività del diritto penale, infatti, non proviene da condanne sommarie di possibili innocenti ma dal corretto ed effettivo accertamento delle responsabilità penali, reso appunto possibile dal rispetto delle garanzie penali e processuali.

Domanda 3: Che valutazione fa degli impatti pratici del modello garantista nella vita della società e sulla vita quotidiana delle persone?

L.F.: Il garantismo, purtroppo, non è popolare. Soprattutto in materia penale l'opinione pubblica, a causa delle campagne politiche in tema di sicurezza, è tendenzialmente colpevolista. Le garanzie non fanno parte della cultura di massa e neppure del senso comune. Gli imputati, secondo l'opinione corrente, non si presumono innocenti ma colpevoli. Il garantismo non 
fa parte del senso comune, né tanto meno della subcultura dei populisti che hanno bisogno, purtroppo, di avventarsi immediatamente su capri espiatori. In breve, esso impopolare, e questo basta al populismo politico per rifiutarlo come un lusso da anime belle. Naturalmente siamo qui di fronte a un paradosso. Il garantismo non è solo un sistema di limiti e vincoli al potere punitivo, sia legislativo che giudiziario, a garanzia delle libertà di tutti da punizioni eccessive o arbitrarie. E' ancor prima un sistema di regole razionali che garantiscono, nella massima misura, l'accertamento plausibile della "verità processuale" e perciò la punizione dei veri colpevoli. Ma è precisamente questa razionalità che non viene accettata né capita da una parte dell'opinione pubblica, diseducata dai media, che aspira al contrario alla giustizia sommaria, tendenzialmente al linciaggio dei sospetti. E anche questo è sufficiente al populismo per offrire rappresentanza a tale concezione e alla conseguente domanda di vendetta.

Il populismo penale è insomma funzionale al populismo politico. Il suo paradigma - il diritto penale del nemico - è in perfetta sintonia con la tendenza del populismo politico a definirsi sulla base di nemici. Tutti i populismi hanno bisogno di legittimarsi attraverso un nemico o meglio più nemici: nemici interni che complottano e nemici esterni come l'Unione Europea o l'Onu; nemici in alto, rappresentati dalle élites, e nemici in basso rappresentati dai migranti e dai soggetti devianti; nemici identificati con i precedenti governi e nemici consistenti nelle opposizioni. Ed è chiaro che l'auto-identificazione delle maggioranze populiste con il popolo sovrano, il razzismo, la paura per i crimini di strada, l'intolleranza del dissenso, il fastidio per il pluralismo sia politico che istituzionale e il vittimismo permanente sono gli ingredienti di questa logica del nemico. L'impatto pratico del garantismo nella società è perciò legato alla critica di queste subculture e dei loro inganni e perciò alla crescita del senso civico a livello di massa, quale solo può provenire da una battaglia culturale a sostegno dei valori costituzionali, dai diritti fondamentali alla pari dignità delle persone.

\section{Domanda 4: Secondo il garantismo, qual è il ruolo della pena e del diritto penale,}

\section{nella società?}

L.F.: Il ruolo delle pene e del diritto penale, secondo la teoria garantista, consiste nella prevenzione e perciò nella minimizzazione della violenza e dell'arbitrio nella società: non soltanto della violenza e dell'arbitrio dei consociati, cioè dei delitti, ma anche della violenza e dell'arbitrio delle istituzioni punitive, cioè delle pene arbitrarie o eccessive. Non dimentichiamo la tesi di Montesquieu secondo cui non soltanto la libertà, ma anche la sicurezza dei cittadini è minacciata soprattutto dalle pene e dalle condanne arbitrarie. 


\section{Domande di GUSTAVO OCTAVIANO DINIZ JUNQUEIRA.}

Specialista in diritto penale presso I'Università di Salamanca/Spagna; Master e dottore in diritto penale presso la PUC-SP/ Brasile; post-doc presso I'Università di Coimbra (Portogallo); professore di diritto penale negli studi di laurea, post-lauream e master presso la facoltà di giurisprudenza della PUC-SP; Docente nel corso di laurea Damásio; Defensor Público nello Stato di San Paolo/Brasile, già direttore della "Escola da Defensoria Pública" per tre mandati .

Preambolo: Il discorso garantista circola in Brasile da decenni. Si tratta di un pensiero 'venerato' che è diventato un importante strumento persuasivo nel dibattito giuridico. Tuttavia, la ripetizione imprudente dell'espressione "garantismo" è stata così frequente da potersi essere gradualmente allontanata dal suo significato originale. Oggi è molto in voga, in Brasile, una corrente di pensiero, capitanata da membri della Magistratura, che si fa chiamare "garantismo penale integrale" e che sostiene di partire dal pensiero di Luigi Ferrajoli per affermare che l'attenzione non dovrebbe essere focalizzata solo sulle garanzie individuali contro il potere punitivo dello Stato, essendo piuttosto necessario "garantire" gli interessi della collettività rispetto al singolo criminale. La conclusione della corrente di pensiero "integrale" è che sarebbe possibile e raccomandabile il sacrificio dei diritti individuali e delle garanzie (come la libertà, il giusto processo, il diritto di difesa, il principio di legalità o di colpevolezza) a favore della tutela penale degli diritti collettivi come la "sicurezza pubblica", la "salute pubblica" e persino la "regolarità della pubblica amministrazione". Secondo la corrente "integrale", l'allentamento delle garanzie non sarebbe un limite di efficacia del garantismo contro le istanze punitive della società, ma una conseguenza del garantismo stesso.

Domanda 5. Si chiede: partendo dalla premessa della massima effettività del garantismo penale, è necessario accettare la flessibilizzazione delle garanzie previste dagli assiomi tradizionali del garantismo in favore della tutela di interessi collettivi come "sicurezza pubblica", "salute pubblica" o "regolarità dell'amministrazione pubblica"? È possibile intendere tale sacrificio come un'evoluzione naturale e prevedibile del garantismo nella difesa degli interessi collettivi?

L.F.: Ho già detto che il "garantismo" non è solo il garantismo penale. Il garantismo è un paradigma teorico valido per tutti i diritti e tutti i beni fondamentali. Ma questo non comporta affatto le conseguenze che, secondo la sua domanda, ne vorrebbero trarre gli esponenti del cosiddetto "garantismo penale integrale". Non si capisce quali vantaggi potrebbero trarre la sicurezza pubblica, la salute pubblica e simili dalle lesioni delle garanzie penali e processuali; mentre sono assolutamente evidenti le lesioni dei diritti e della sicurezza dei cittadini che da tali lesioni proverrebbero. 


\section{Domande di JOÃO PAULO MARTINELLI.}

Professore presso l'Istituto di Diritto Pubblico di San Paolo (IDP / SP) e I'Università Federale Fluminense (RJ), Brasile. Post-doc in diritti umani presso I'Università di Coimbra / Portogallo. Master e dottore in diritto penale presso la Facoltà di Giurisprudenza della Università di San Paolo (USP) Brasile. Già membro della commissione giovani penalisti Dell'associazione Internazionale di Diritto Penale (AIDP). Ė stato ricercatore presso I'Università di Monaco (Germania) e l'Università della California (UC Davis, USA). Ha studiato post-lauream diritto penale (teoria del crimine) presso I'Università di Salamanca (Spagna). Specializzato in diritto penale internazionale dall'International Institute of Higher Studies in Criminal Sciences (ISISC). Coordinatore di accordi dell'Istituto Brasiliano di Ccienze Criminali (IBCCRIM / Brasile). Membro della Commissione di diritto penale economico dell'Ordine Avvocati Brasiliani (OAB/SP).

Domanda 6. In Brasile, è molto comune per il giudice penale interrogare i testimoni e porre domande dettagliate, particolarmente specifiche, come se volesse produrre prove contro l'imputato. In un autentico sistema accusatorio il giudice può interrogare i testimoni?

L.F.: Nel sistema accusatorio il giudice deve mantenere un atteggiamento di totale terzietà e imparzialità. Non gli è quindi consentito di interrogare i testimoni che è quanto invece compete alle parti del processo. Tanto meno può formare prove contro l'imputato. Prove e controprove vanno formate nel pubblico dibattimento e nel contraddittorio, su iniziativa della pubblica accusa e della difesa.

Domanda 7. La dottrina brasiliana tradizionale, e anche la giurisprudenza, difendono il principio della "verità materiale", secondo la quale il giudice deve cercare la verità dei fatti per formare il suo convincimento. Questa posizione conferisce al giudice un ruolo di protagonista, come se fosse parte del processo. Qual è la sua opinione sul cosiddetto principio della "verità materiale"?

L.F.: La sola verità che legittima il processo è la verità processuale, acquisita attraverso il rigoroso rispetto di tutte le garanzie del corretto processo. Non condivido affatto questo cosiddetto principio della verità materiale che, al di là delle intenzioni, rischia sempre di risolversi in una legittimazione dell'arbitrio. Naturalmente i giudici per primi devono essere consapevoli del carattere probabilistico della verità fattuale e opinabile della verità giuridica. Il dubbio è la prima regola della deontologia giudiziaria.

Domanda 8. Recentemente, un giudice di Cassazione brasiliano ha subito un procedimento amministrativo per aver concesso la liberazione dei detenuti che erano in detenzione oltre il tempo previsto nella decisione di condanna. L'argomento principale della Corte era la violazione del principio di collegialità, poiché era membro di una camera criminale. Esiste davvero un principio di collegialità? Una decisione che revoca un arresto illegale può essere resa monocratica da un membro del tribunale?

L.F.: Non conosco la procedura brasiliana, né quindi la distribuzione delle competenze determinata dal "principio di collegialità" richiamato dalla domanda. Se il giudice sottoposto a "procedimento amministrativo per aver concesso la liberazione dei detenuti che erano in detenzione 
oltre il tempo previsto nella decisione di condanna" aveva la competenza all'emanazione di tale provvedimento, allora il suo atto era non soltanto lecito, ma doveroso, e il procedimento amministrativo è stato del tutto infondato.

Domanda 9. Quale dovrebbe essere il ruolo della vittima nello sviluppo del processo penale? La vittima può attuare come assistente dell'accusa o questo ruolo dovrebbe essere esclusivo del titolare dell azione penale?

L.F.: La vittima è titolare soltanto del diritto, in caso di condanna, al risarcimento dei danni subiti. Quanto all'aspetto penale del processo, la vittima è legittimamente interessata non già alla condanna quale che sia, bensì all'accertamento della verità processuale, cioè all'accertamento della responsabilità dei veri colpevoli, e quindi al rispetto delle garanzie processuali che come ho già detto sono anzitutto garanzie di verità.

\section{Domande di PASQUALE BRONZO.}

Professore Associato di diritto processuale penale presso Sapienza Università di Roma.

Domanda 10. Nella sua idea di riforma del sistema penale c'è una riduzione delle incriminazioni, anche attraverso una depenalizzazione, tra gli altri, dei reati che non hanno un'autentica efficacia di deterrenza, riguardando «comportamenti commessi comunque e che la pena ha il solo effetto di rendere clandestini» (Ferrajoli, L., Dei diritti e delle garanzie, Il Mulino, 2013, p. 26). Tra le incriminazioni di quest'ultimo tipo fa menzione del reato di immigrazione irregolare (art. 10-bis decreto legislativo 25 luglio 1998, n. 286) introdotto in Italia dal 2009, che si è dimostrato privo di qualsiasi effetto dissuasivo e capace solo di sospingere i migranti irregolari nell'illegalità, contribuendo a renderli facile preda delle organizzazioni criminali come la mafia. Un caso paradigmatico di uso simbolico del diritto penale: lo straniero entrato illegalmente - che non può essere arrestato, trattandosi di contravvenzione - viene processato dopo mesi o anni (solitamente in sua assenza), condannato ad una pena pecuniaria che non viene mai riscossa (mentre il processo costa alla collettività, sia in termini di personale giudiziario, sia perché lo straniero indigente ha diritto ad essere difeso a spese dello Stato). Questa incriminazione - in sé inutile - è stata introdotta solo per mantenere la 'rassicurante' possibilità di espulsione coattiva dell'irregolare (senza concessione di termini per la partenza volontaria) che una direttiva europea dal 2008 ci permette solo ove l'espulsione sia sanzione penale o conseguenza di una sentenza penale. Così accade che se durante il processo si riesce ad eseguire l'espulsione in via amministrativa, il processo si blocca, altrimenti arriva la pena pecuniaria, sostituibile dall'espulsione ove possibile. Senonché, questo particolare tipo di espulsione penale risulta quasi sempre impossibile, per gli stessi motivi per cui è impossibile quella amministrativa, conducendo 
alla disapplicazione pressoché generalizzata della norma (anzi, la disapplicano la maggioranza degli uffici giudiziari, determinando anche inammissibili disomogeneità). Crede che il sistema penale "espansionista" del Brasile, da sempre incline alla legislazione simbolica, $e$ oggi afflitto da problemi di immigrazione massiccia come quella proveniente dal Venezuela, possa far tesoro dell'esempio negativamente istruttivo del nostro reato di 'clandestinità'?

L.F.: Il diritto penale, secondo il suo modello garantista, non ha la funzione di declamare principi politici, ma soltanto di prevenire danni ingiusti. Norme penali destinate a rimanere ineffettive - come quelle che penalizzano l'aborto o l'immigrazione clandestina o l'uso personale di droghe - al di là di ciò che pensiamo in ordine alla loro illegittimità morale e politica, sono norme ingiustificate semplicemente perché inidonee a prevenire i fatti da esse penalizzati. Di più, esse sono sempre tendenzialmente criminogene, dato che il loro effetto principale è quello di rendere clandestini i comportamenti da esse previsti e proibiti. La loro ineffettività, documentata dalla sua domanda, è una conferma della loro inutilità o peggio della loro dannosità.

Domanda 11. Il Brasile vive oggi un grave problema di sovraffollamento carcerario (al quarto posto nel mondo, per popolazione penitenziaria). Nella sua idea di riforma della giustizia in Italia c'è una forte riduzione del carcere, da realizzare non solo diminuendo la durata delle pene edittali, ma anche introducendo pene detentive non carcerarie per reati di modesta gravità, con contenuti simili a quelli delle attuali misure alternative alla detenzione, ma applicate direttamente in sentenza, dal giudice della cognizione (Ferrajoli, L., Dei diritti e delle garanzie, Il Mulino, 2013, p. 28). In Italia siamo andati molto vicini all'approvare una riforma simile nel 2014 , attraverso un nuovo catalogo di sanzioni: prescrizioni, detenzione domiciliare, $\mathrm{e}$ in eventuale aggiunta a quest'ultima, lavoro di pubblica utilità; con una completa riconfigurazione del lavoro 'penale' che, nato come pena, e poi espediente per inasprire il carcere, e quindi diventato un mezzo per dare senso al carcere, tornerebbe ad essere pena, ma fuori e senza il carcere. Pensa che questo modello sia realizzabile anche in un sistema caratterizzato da una vera e propria carcerazione di massa come quello brasiliano?

L.F.: Il sovraffollamento è di per sé un trattamento contrario al senso di umanità che, in quanto tale, è illegittimo e non deve comunque essere consentito. Quanto alla carcerazione di massa, in Brasile come negli Stati Uniti, essa è sempre il segno inequivoco del carattere discriminatorio e classista del diritto penale di questi paesi. Tanto più in questi paesi sarebbero perciò necessari imponenti processi di de-penalizzazione e de-carcerizzazione. Sono anni che propongo da un lato la riduzione della durata massima della pena a 15 o al massimo a 20 anni e, dall'altro, la previsione, per tutti i reati non gravi, di un ampio ventaglio di sanzioni penali alternative alla reclusione. Aggiungo che una vera politica anti-criminale, che abbia realmente 
a cuore la sicurezza, dovrebbe essere, ben più che una politica penale, una politica sociale, in grado di ridurre le cause economiche e materiali della criminalità di strada e di sussistenza e ovviamente più difficile e impegnativa degli inasprimenti demagogici delle pene.

Domanda 12. Lei è uno dei primi e principali sostenitori del principio della 'riserva di codice' come strumento per restituire certezza, conoscibilità, e sistematicità ad un diritto penale che - soprattutto a causa di una smisurata produzione normativa e della sempre più evidente 'decodificazione' - ha perduto certezza, conoscibilità, coerenza interna; tanto che - come lei ricorda (Ferrajoli, L., Crisi della legalità penale e giurisdizione. Una proposta: la riserva di codice, in Legalità e giurisdizione. Le garanzie penali tra incertezze del presente ed ipotesi del futuro, Padova, 2001, 27 ss) - una sentenza della Corte costituzionale del 1988 ha dovuto affermare che il principio per cui 'l'ignoranza della legge non scusa', non vale quando l'ignoranza è inevitabile.

In Italia, l'anno scorso, questo principio ha trovato attuazione - seppure con importanti limiti - nel decreto legislativo $1^{\circ}$ marzo 2018, n. 21, che ha introdotto (nel codice penale, e cioè in una legge ordinaria) una previsione in base alla quale cui «nuove disposizioni che prevedono reati possono essere introdotte nell'ordinamento solo se modificano il codice penale ovvero sono inserite in leggi che disciplinano in modo organico la materia». Lei ritiene che nella realtà attuale, caratterizzata - a livello globale - da un diritto penale sempre più 'multilivello', e da un impiego sempre più frequente di fattispecie penali come norme 'di chiusura' di determinate discipline di settore, vi siano le condizioni politico-culturali per osservare il principio della riserva di codice così come originariamente concepito, o pensa che sia inevitabile accontentarsi - secondo l'impostazione di quel decreto - di una razionalizzazione della produzione legislativa che, pur senza eliminare il diritto penale extra-codicem, richieda al legislatore di creare quante più leggi organiche possibili?

L.F.: Ho avanzato da vari decenni la proposta della riserva di codice, che è una riserva di legge penale rafforzata e idonea ad assicurare al diritto penale conoscibilità ancor prima che certezza. E' una proposta che è diventata ancor più necessaria ed urgente in presenza della complicazione del sistema delle fonti, e addirittura, come lei dice, di un diritto penale "multilivello", il quale a sua volta dovrebbe sottostare a una riserva del medesimo tipo. Si tratta di una banale garanzia di razionalità e conoscibilità, che può essere avversata soltanto dalla demagogia populista, la quale si vedrebbe privata dell'uso congiunturale e propagandistico del diritto penale. 


\section{LUIGI FERRAJOLI - INTERVISTA \\ GARANTISMO E CONSTITUZIONALISMO (parte seconda)}

\section{Domande di ENZO BELLO.}

Dottore in giurisprudenza presso l'Universitá dello Stato di Rio de Janeiro (UERJ), Brasile. Post-doc in diritto presso l'Università del Vale do Rio dos Sinos (UNISINOS), Brasile, e servizio sociale presso I'Universitá Federale di Rio de Janeiro (UFRJ), Brasile. Professore aggiunto presso la Facoltà di Giurisprudenza e nel programma di post-lauream in diritto costituzionale presso l'Universitá Federale Fluminense (UFF), Brasile. Direttore scientifico della Rivista Culturas Jurídicas (www.culturasjuridicas.uff.br).

Domanda 13. Conosce il cosiddetto "nuovo costituzionalismo latinoamericano", rappresentato dalle costituzioni di Venezuela (1999), Ecuador (2008) e Bolivia (2009)?

Luigi Ferrajoli (L.F.): Ne ho una conoscenza alquanto sommaria. Ho parlato più volte, a proposito di queste costituzioni e soprattutto di quella brasiliana, di "costituzionalismo di terza generazione". Queste costituzioni sono costituzioni lunghe, sul modello della costituzione portoghese e, soprattutto, hanno introdotto nuovi diritti, come i diritti all'acqua, all'accesso alle comunicazioni e alle tecnologie informatiche, all'uso del gas e dell'elettricità nelle costituzioni dell'Ecuador e della Bolivia. Ma ho qualche perplessità sulla categoria “diritti della natura", che è evidentemente una figura metaforica, presente nell'articolo 71 della Costituzione dell'Ecuador. Più appropriata ed efficace sarebbe stata la qualificazione costituzionale come beni pubblici e demaniali, sottratti al mercato e alla disponibilità della politica, di beni naturali come l'acqua, l'aria, i fiumi, i mari e in generale quelle che Gaio configurò come "res communes omnium".

Domanda 14. Legato a questo, ha avuto contatto con dottrina latinoamericana di Diritto Costituzionale, Teoria del Diritto e/o Diritti Umani? In tal caso, vede affinità in relazione alla sua opera, legata al contesto europeo?

L.F.: Vedo affinità in qualunque orientamento teorico diretto a rafforzare le garanzie dei diritti umani. In particolare ho più volte sottolineato il valore di molte nuove garanzie inventate dal costituzionalismo latino-americamo: dai Tribunali elettorali e le altre istituzioni elettorali di garanzia introdotti in Messico al pubblico ministero di difesa introdotto in Argentina e in molti altri paesi latino-americani, dai vincoli di bilancio che in Brasile impongono la destinazione alla garanzia dei diritti alla salute e all'istruzione di quote minime della spesa pubblica alle funzioni di garanzia di tali diritti affidate, sempre in Brasile, agli uffici del Pubblico Ministero, fino alla previsione, anch'essa in Brasile, di un Procuratore presso il Tribunale Costituzionale abilitato, insieme ad altri soggetti (minoranze parlamentari, presidente, governatori e altri), a promuovere l'azione di incostituzionalità

Domanda 15. Qual è la sua valutazione sulla relazione tra i piani teorici e pratici del neo-costituzionalismo / post-positivismo e neoliberismo?

L.F.: Sono contrario a tutti gli orientamenti teorici di tipo anti-giuspositivista, siano essi post-positivisti o a-positivisti o giusnaturalisti. Esiste a mio parere un nesso strumentale, 
generalmente trascurato, tra positivismo giuridico, garantismo e democrazia. Il positivismo giuridico forma il presupposto necessario del garantismo, che è l'altra faccia del costituzionalismo e perciò della democrazia costituzionale. La positivizzazione delle regole, formali e sostanziali, sulla produzione giuridica si è infatti rivelata come la tecnica che ha reso possibile la democratizzazione sia delle forme che dei contenuti del diritto. E' questa una tesi descrittiva, indipendente delle nostre opzioni filosofiche o politiche di tipo giuscostituzionalista o giuspositivista o giusnaturalista, oppure realista o liberista. Ovviamente né il costituzionalismo né tanto meno il positivismo giuridico implicano la democrazia e il garantismo. E' però innegabile che la positivizzazione delle norme formali sulle forme della produzione giuridica, operata con il primo giuspositivismo dello stato legislativo di diritto, ha permesso di stipulare, quali norme di diritto positivo, le regole formali della democrazia politica, dal suffragio universale alla rappresentanza politica, dalla separazione dei poteri al controllo di costituzionalità sulle leggi. Per altro verso, la positivizzazione delle norme sostanziali sui contenuti della produzione legislativa, operata con il secondo giuspositivismo dello stato costituzionale di diritto, ha permesso di stipulare, quali norme positive di rango sopraordinato a qualunque altra, le regole della democrazia sostanziale, e cioè i limiti e i vincoli imposti alle maggioranze nella forma dei diritti fondamentali delle persone in esse stabiliti.

\section{Domande di PIERRE MOUSSERON.}

Professore di diritto dell'imprese presso la Facoltà di Giurisprudenza di Montpellier - Francia. Presidente dell'Instituto degli Usi mercantili (Institut des usages-Montpellier-Francia).

Domanda 16. L'Unione europea ha adottato provvedimenti legislativi in materia penale, in particolare per quanto riguarda la lotta contro il riciclaggio di denaro. La Corte di giustizia dell'Unione europea ha pronunciato decisioni in materia penale in base alle quali sono stati affievoliti alcuni principi consolidati, come quello di colpevolezza (CJUE 5 marzo 2015, C-343-13). Non pensa che l'Unione europea stia superando le proprie prerogative, con tali azioni?

L.F.: Il principio di colpevolezza è un principio garantista assolutamente basilare. L'affievolimento di tale principio va criticato di per sé, e non già in quanto la legislazione penale dovrebbe rimanere estranea alle competenze dell'Unione Europea. Al contrario ho sempre sostenuto che il processo di integrazione dell'Unione richieda, tra le altre innovazioni istituzionali - prima fra tutte la forma politica federale - l'unificazione giuridica di gran parte della legislazione di base. Non ha senso l'esistenza in Europa di 27 codici civili, di 27 codici penali e di altrettanti di procedura sostanzialmente simili. L'unificazione giuridica, in particolare della codificazione penale, sarebbe un sicuro fattore di integrazione, di crescita dell'uguaglianza e, insieme, di effettività del diritto penale. 
Domanda 17. Nel mio centro di studi a Montpellier la validità degli usi quali fonte del diritto in rapporto alle regole generali dell'Unione Europea, per lo più riguardo alle persone giuridiche e alle associazioni tradizionali di produttori e venditori (di vino, ad esempio), è oggetto di un interesse molto forte e risalente. In base alla sua esperienza di difesa dei diritti individuali e delle garanzie, cosa pensa dei diritti di tali soggetti giuridici? Concentrandosi sul nostro argomento degli "usi normativi", giacché le regolamentazioni formali possono essere viste come fonti di aumento della burocrazia, pensa che regole non formali come gli usi potrebbero essere, almeno in alcune aree, una valida alternativa a un processo senza fine di 'formalizzazione' legale dei diritti individuali?

L.F.: Gli usi e le consuetudini sono certamente fonti informali di diritto, che stanno riconquistando una rilevanza che sembrava cessata in un non lontano passato. Continuo tuttavia a pensare che la loro legittimità è condizionata alla loro non contrarietà alla legge. Temo inoltre che difficilmente essi possano essere "una valida alternativa" alla formalizzazione legale dei diritti fondamentali.

Domande di NATALIA GASPAR.

Avvocata e Professoressa di diritti umani, diritto penale dell'impresa e diritto econômico presso l'Universidad Autonoma de Puebla BUAP/México.

Domanda 18. L'arrivo della sinistra al potere in Messico nel 2018 è visto come un passaggio di trasformazione verso la democrazia all'interno del modello neo-costituzionalista e garantista?

L.F.: Spero sinceramente che il nuovo Presidente messicano riesca a promuovere una crescita democratica del Messico, attraverso una maggiore garanzia dei diritti sociali e perciò una riduzione della povertà e delle enormi disuguaglianze. Aggiungo che un'effettiva crescita della democrazia può essere solo ostacolata dalle tentazioni populiste, sempre tendenzialmente in contrasto con il paradigma garantista.

Domanda 19. Ritiene che il Messico, con una costituzione del 1917 e più di 600 riforme, oltre alle riforme in materia economica nel 2013 (telecomunicazioni, energia, concorrenza finanziaria ed economica), sia in linea con il neo-costituzionalismo?

L.F.:“Neo-costituzionalismo", a mio parere, è un'espressione equivoca e talora fuorviante. Preferisco parlare di costituzionalismo tout court, oppure di costituzionalismo giuridico o garantista, generato dalle odierne costituzioni rigide, in opposizione al costituzionalismo politico che invece è una dottrina politica ben più antica, che si basa sul governo delle leggi in opposizione al governo degli uomini. Quanto alla vecchia Costituzione messicana, il vero problema, ben più che la sua riforma, è l'attuazione dei tanti diritti fondamentali in essa stabiliti, a cominciare dai diritti sociali alla salute, all'istruzione e alla sussistenza, attraverso l'introduzione per via legislativa di idonee garanzie e di adeguate funzioni e istituzioni di garanzia. 


\section{Domande di JOSÉ ANTONIO SIQUEIRA PONTES.}

Master e dottore in filosofia e teoria generale del diritto presso I'Università di San Paolo (USP), Brasile. Ricercatore presso le Facoltà di Campinas (FACAMP), Brasile. Coordinatore del gruppo di ricerca sui fondamenti economici e sociologici dell'integrità delle imprese (compliance) / FACAMP.

Preambolo: Nelle sue opere sul costituzionalismo come manifestazione del garantismo, almeno a partire dagli anni 2000, Lei presenta un senso di realtà sociologica evidenziando le crisi dei sistemi di protezione dei diritti e i necessari cambiamenti nel diritto locale e globale per superare le difficoltà. Sottolineo qui l'importanza che Lei da alla sfera pubblica, ai poteri dello Stato in contrapposizione agli interessi privati di individui e corporazioni. Questa sfera pubblica è la base concettuale per l'effettiva garanzia di un insieme di diritti, come l'accesso al reddito minimo, la salute e l'istruzione gratuite, l'ambiente sano e così via. Lei spesso associa questi obiettivi di cittadinanza di base a obiettivi di diritto penale minimo, cioè riduzione della criminalità di massa e dell'incarcerazione crescente dei poveri. Ad ogni modo, se ho capito bene, difende una rete concettuale che collega la realizzazione dei diritti fondamentali a una forte sfera pubblica capace di contrastare gli interessi privati, attraverso la necessaria espansione delle varie funzioni statali nazionali e il rafforzamento delle organizzazioni internazionali, verso una nuova forma del costituzionalismo globale. Parallelamente, notiamo che organizzazioni internazionali come l'OCSE, il FMI, la Banca mondiale, lo IOSCO, il Comitato di Basilea, tra gli altri, mostrano recenti cambiamenti nel modello di “diritti” derivanti dall'ambito internazionale come condizione di buona "governance privata". Questa idea di governance appare come una manifestazione di un "nuovo ordine liberale", quindi governata in armonia con interessi commerciali privati come trasparenza, integrità e riduzione del rischio. Tuttavia, la logica della governance è sempre più legata alla gestione degli Stati, da un lato, attraverso l'efficienza, la salute fiscale, il taglio dei costi e, dall'altro, la delega di poteri e funzioni pubbliche all'ambiente privato (autoregolamentazione, ecc.), che è un altro aspetto del motto "privatizzazione". D'altra parte, questo fenomeno globale ha un altro aspetto interessante: queste stesse organizzazioni para-governative internazionali (OCSE, FMI, Banca Mondiale, IOSCO, Basilea) sembrano legiferare nel padrone di soft law, definendo linee guida internazionali che sono una fonte complementare del diritto degli Stati (leggi penali, civili e amministrative) molte delle quali sono più cogenti di molte leggi nazionali nella pratica. Sembrano rafforzare in qualche misura le possibilità future di una comunità pubblica internazionale e l'ONU allinea sempre più i suoi programmi di diritti fondamentali e di sostenibilità (Agenda 2030) con il discorso della governance, la trasparenza nella lotta alla corruzione, il terrorismo e il riciclaggio di denaro.

Le domande, in questo senso, partono dalle relazioni e separazioni tra sfera pubblica e sfera privata e riguardano il futuro dei diritti per le generazioni future:

Domanda 20. Qual è la sua analisi di questi movimenti relativamente recenti? Esiste la possibilità che questo "ideale di compliance internazionale" rafforzi la nozione di collettività, 
di comunità internazionale a sostegno o la graduale sostituzione dei costituzionalismi locali con uno spirito pubblico globale cosmopolita che ha come obiettivo la realizzazione di diritti costituzionali fondamentali o, al contrario, è un sintomo attuale dei nostri fallimenti nell'affermare la sfera pubblica a livello locale o internazionale?

L.F.: Con una formula sommaria, dirò che il garantismo, cioè l'insieme delle garanzie idonee a tutelare e a soddisfare i diritti fondamentali, richiede da un lato uno stato penale minimo (un "diritto penale minimo" come ho chiamato il modello garantista del diritto penale) e, dall'altro, uno stato sociale massimo: in altre parole, un passo indietro della sfera pubblica a tutela delle libertà fondamentali e un suo passo avanti a garanzia dei diritti sociali. Che è esattamente l'opposto di quanto sta accadendo nelle nostre democrazie, nelle quali si è dato vita a un diritto penale massimo in accordo con le demagogie populiste e a uno stato sociale minimo in ottemperanza alle istanze liberiste. In breve, sono contrario a tutte le privatizzazioni di servizi pubblici e di pubbliche funzioni di garanzia che rischiano sempre di trasformare in diritti patrimoniali quelli che le nostre costituzioni hanno configurato come diritti sociali fondamentali. Un tratto distintivo dei diritti fondamentali è infatti il carattere gratuito delle loro garanzie - in particolare delle prestazioni dovute in ottemperanza dei diritti sociali - conseguente alla loro forma universale, quali diritti di tutti e fondamento dell'uguaglianza. E mi riesce difficile ipotizzare una scuola privata gratuita o una sanità privata parimenti gratuita.

Domanda 21. Il concetto di governance è per alcuni un fenomeno originale del calcolo e dell'efficienza, la manifestazione attuale e irrefrenabile di sviluppo commerciale moderno (Supiot A., La gouvernance par les nombres). Emerge importanti conseguenze, come l'idea di JURIMETRICS o l'uso di misure statistiche per riflettere sulla razionalità del diritto. Come un esempio positivo possiamo citare la materia attuale sui giornali brasiliani dando notizia che la mancanza di criteri giuridici chiari, la polizia decide chi è un utente e chi è uno spacciatore di droga (https://noticias.uol.com.br/ultimas-noticias/ agencia-estado/2019/03/31/sem-lei-que-cite-quantidades-policia-decide-quem-sera-preso-por-trafico.htm ). Informatizzazione della vita e ampio accesso ai big data, l'uso di statistiche e metriche hanno, però, un altro aspetto: il dominio della vita digitale sulla soggettività, ora mediata da "social network digitali". Questa realtà infrastrutturale riguarda le professioni, essendo la manifestazione più chiara nelle professioni giuridiche la sostituizione dei processi fisici da processi digitali, le udienze virtualizzate in teleconferenza e l'acesso di supercomputer come Watson (IBM) o Coin (JP Morgan) a basi infinite di leggi, regolamenti, contratti e raccolte di giurisprudenza. In nome della suddetta efficienza e governance, i poteri di giustizia (magistratura, pubblico ministero, difensori, avvocati) sentono i loro effetti: ridurre le persone e aumentare i processi in quantità sovrumane può 
essere gestito solo da grandi sistemi informatici. Molti professionisti in Brasile lamentano già l'assenza di mediazione umana nella realizzazione dei diritti fondamentali dei cittadini nei loro rapporti con lo Stato, tra cui il diritto di essere ascoltati, di leggere le loro petizioni e altre garanzie di giusto processo. Come Lei vede questo conflitto tra efficienza digitale e garantismo nel senso umano, con processi che coinvolgono le persone, una manifestazione delle garanzie classiche del "giudice naturale" o "promotore naturale" e altri diritti che costituiscono "reale" piuttosto che "virtuale" giustizia e Stato? La stessa era digitale che ci consente di vedere gravi distorsioni nella disuguaglianza di trattamento tra quelli che usano droghe, rivela una tendenza ad allontanare il giudice dai suoi imputati, dallo stato dei suoi cittadini, dalla gente dei suoi rappresentanti?

L.F.: Anche "governance", a me pare, è un concetto ambiguo e sfuggente, che non fa parte del lessico garantista. Quanto ai processi digitali, agli algoritmi e alla sostituzione degli apparati della sfera pubblica con apparati interamente informatizzati, essi mi sembrano tutti inquietanti e, se non sottoposti a limiti e a garanzie, altresì pericolosi. Questi algoritmi, questi automatismi con cui ci si illude di sostituire la discrezionalità amministrativa con una sorta efficienza oggettiva e neutrale nascondono una minaccia alle garanzie dei diritti. Essi, infatti, non sono affatto neutrali. Sono necessariamente costruiti sulla base della scelta, niente affatto avalutativa, di uno o più parametri di valutazione contrabbandati come oggettivi e sul rifiuto o l'irrilevanza di altri criteri o parametri. Producono perciò, inevitabilmente, una deresponsabilizzazione e un occultamento dei poteri per il loro tramite esercitati e una loro legittimazione come poteri "tecnici".

Domanda 22. La diagnosi della crisi politica globale si trova anche nella sua opera più recente, Manifesto per l'uguaglianza (2018). Le tendenze che Lei descrive, nell'analisi del rapporto tra legge ed economia sono presenti in molti paesi, in particolare quelli latinoamericani come il "nuovo ordine liberale". Ha detto: «Queste politiche hanno dapprima determinato la crisi, e poi l'hanno aggravata proponendo come terapie le stesse misure dalle quali la crisi è stata provocata: la deregolazione delle relazioni di mercato e in particolare delle attività finanziarie; la riduzione delle imposte sui ricchi, a cominciare da quelle sulle successioni, e perciò delle entrate fiscali; la libera circolazione dei capitali, grazie alla quale questi possono evadere il fisco trasferendosi nei paradisi fiscali; i tagli alla spesa pubblica nella sanità e nell'istruzione; gli abbassamenti dei salari e delle pensioni; la precarizzazione del lavoro e la demolizione del diritto e dei diritti dei lavoratori; la sottomissione alla finanza, a causa della quale i governi hanno in questi anni stanziato migliaia di miliardi di euro per salvare le banche, dopo averle privatizzate e consentito loro di giocare d'azzardo sui mercati, ma non sono riusciti a trovare poche decine di miliardi 
per salvare la Grecia dal disastro economico e sociale» (Ferrajoli, L., Manifesto per l'uguaglianza, Editori Laterza, 2018, iBooks.) È nello stesso capitolo che Lei fa una critica di passaggio al concetto di Law and Economics e cita l'abbandono delle politiche keynesiane, dando come esempio le politiche di intervento fatte dal governo Lula in Brasile e il loro relativo successo nel ridurre la miseria.

Lei sembra riflettere un movimento che è a volte tacitamente presente in tutti i fondamenti delle politiche pubbliche, nel dibattito politico ed economico che sta alla base di ogni reale base di effettività dei diritti fondamentali, ambientali, sociali e umani, o di qualsiasi diritto. $\grave{E}$ un movimento per rafforzare la sfera pubblica, lo Stato, per affrontare la deregolamentazione privata, finanziaria ed economica.

Possiamo dire che questa dottrina è una condizione reale e sostanziale per le varie dimensioni del costituzionalismo, del garantismo che Lei difende per decenni? Possiamo dire che si tratta di un movimento di "Diritto ed Economia Politica" per affrontare la Law and Economics? Come potrebbe definire questa dottrina? Le generazioni future avranno le condizioni per conquistare a livello internazionale (ONU, FMI, OCSE, Banca Mondiale, ecc.) un consenso sul fatto che non ci saranno diritti fondamentali senza politiche economiche di Stato interventivi, di linea keynesiana, globalmente sostenibili, attraverso poteri pubblici forti, presenti ed attuanti?

L.F.: La costruzione della sfera pubblica, ripeto, è stata la grande conquista della modernità giuridica e politica. Naturalmente la sfera pubblica può anche essere - ed è stata, e continua in molti paesi ad essere - una sfera pubblica illiberale o antisociale e perciò anti-democratica. Le costituzioni del secondo Novecento, pur con i loro limiti e difetti, hanno tuttavia disegnato e imposto la costruzione di una sfera pubblica democratica, finalizzata alla garanzia delle varie classi di diritti fondamentali in esse stabiliti. Oggi stiamo assistendo a un'aggressione a questa sfera pubblica democratica, dall'alto e dal basso. In Italia, ma anche in molte altre democrazie occidentali, stiamo assistendo a una perversa e paradossale alleanza tra populismi e liberismo. Le politiche liberiste hanno demolito il diritto del lavoro e le sue garanzie e, conseguentemente, le vecchie forme di soggettività collettiva basate sull'uguaglianza e su lotte comuni per comuni diritti. Espressioni come movimento operaio e classe operaia sono andate fuori uso perché è venuta meno, con la moltiplicazione, la differenziazione e la precarizzazione dei rapporti di lavoro, l'uguaglianza nei diritti tra i lavoratori, i quali, anziché solidarizzare in lotte comuni, sono costretti a entrare in competizione tra loro. In questo modo hanno creato le basi sociali degli odierni populismi, che con le loro politiche sulla sicurezza alimentano paure e razzismi quali fattori di nuove soggettività politiche collettive, basate sulla difesa di supposte identità collettive - "prima gli italiani" e simili - da supposte identità nemiche e sull'intolleranza 
per le differenze. A sua volta i populismi ricambiano il favore loro procurato dalla disintegrazione liberista delle vecchie soggettività sociali con la loro opposizione, in nome di un'illusoria sovranità nazionale, alla costruzione di una sfera pubblica sovranazionale, di tipo globale o quanto meno europeo: che è esattamente l'assenza di regole e controlli all'altezza dei mercati, assolutamente funzionale alle politiche liberiste. Infine, populismi sovranisti e liberismo sono accomunati dall'insofferenza per limiti e vincoli costituzionali, generata da un lato dall'ideologia populista della volontà popolare incarnata dalla maggioranza come unica fonte di legittimazione politica e, dall'altro, dall'ideologia liberista del mercato come luogo delle libertà fondamentali che non ammettono limiti né controlli. E' poi evidente che delle due sovranità, quella popolare e quella dei mercati, la seconda è destinata a prevalere sulla prima, se non altro per l'asimmetria tra il carattere globale dell'economia e della finanza e il carattere prevalentemente statale della politica e del diritto. Diritti umani, solidarietà e dignità della persona - gran parte del vocabolario costituzionale - sono del resto sconosciuti sia al linguaggio liberista che a quello populista.

\section{LUIGI FERRAJOLI - INTERVISTA INSEGNARE LA CITTADINANZA (parte terza)}

\section{Domande di GUILHERME MADEIRA DEZEM.}

Master e dottore in diritto processuale penale presso I'Università di San Paolo (USP), Brasile. Professore di diritto processuale penale all'Università presbiteriana di Mackenzie, Brasile. Giudice di diritto a San Paolo, Brasile.

Domanda 23 - Caro professore, il Brasile sta attraversando momenti delicati: i valori democratici e la protezione dei diritti umani non trovano aderenza alla popolazione generale o agli studenti di diritto. Dove noi, gli insegnanti di giurisprudenza, siamo sbagliati che non siamo stati capaci di trasmettere questi valori agli studenti? Cosa possiamo fare?

Luigi Ferrajoli (L.F.): Potete fare moltissimo. Innanzitutto mostrando il ruolo che la scienza giuridica, le cui singole discipline appaiono di solito puramente tecniche e certamente non appassionanti, può svolgere nella critica del diritto illegittimo, cioè dell'illegittimo esercizio dei poteri, sia pubblici che privati. In questo consiste il maggior fascino della scienza giuridica quale è stata rifondata dalle odierne costituzioni rigide e che l'insegnamento ha il compito di rivelare. Il diritto può essere uno strumento potente di costruzione della democrazia, tanto quanto è in grado di operare come sistema di limiti e vincoli nei confronti dei poteri, altrimenti selvaggi, e di garantire i diritti fondamentali di tutti. Purtroppo non è solo il Brasile che sta attraversando una crisi della democrazia. Anche l'Italia, gran parte dell'Europa, perfino 
gli Stati Uniti stanno vivendo analoghe crisi. E' importante mostrare agli studenti che in queste crisi involutive non c'è nulla di naturale o di inevitabile; che i sistemi politici sono come li costruiamo sul piano giuridico, come li immaginiamo e li vogliamo sul piano politico e, prima ancora, come li pensiamo sul piano teorico. Per questo essi dipendono dalla - e sollecitano laresponsabilità di tutti noi, a cominciare da noi giuristi.

Domanda 24. Ci rendiamo conto che nel mondo c'è un'onda che respinge i diritti e le garanzie, in particolare quelli noti come prima generazione o dimensione. Viktor Orban, Matteo Salvini, Donald Trump e, in Brasile, Jair Bolsonaro sono il volto visibile di questo movimento. Apparentemente è un movimento a pendolo. Quanto ci possiamo aspettare che duri questo movimento? Qual è la migliore posizione che noi docenti dovremmo avere?

L.F.: Si tratta di movimenti che crescono soprattutto nel vuoto di cultura politica e giuridica: un vuoto che finisce facilmente per essere colmato da paure, xenofobie, rancori, recriminazioni, egoismi individuali e nazionali alimentati dalle demagogie irresponsabili e spesso dalle falsificazioni e mistificazioni della realtà. La migliore risposta, ripeto, è lo sviluppo del senso civico, della cultura giuridica e politica ed anche l'invenzione di nuove tecniche o forme di garanzia in grado di ridurre i poteri, altrimenti, come sempre, selvaggi e distruttivi.

\section{Domande di DANIEL GUIMARÃES ZVEIBIL.}

Master e dottore in diritto processuale presso I'Università di San Paolo (USP), Brasile, su temi di diritto processuale costituzionali. Discente di fondamenti di psicoanalisi e la sua pratica clinica presso I'Istituto Sedes Sapientiae, Brasile. Professore di diritto processuale. Membro del CEAPRO (Centro di studi avanzati della procedura / Centro de Estudos Avançados de Processo) e IBDP (Istituto brasiliano di diritto processuale / Instituto Brasileiro de Dijreito Processual). Defensor Público dello Stato di San Paolo con attribuzioni nella prima Giuria della capitale.

Preambolo: Se si considera la prospettiva del secondo significato di "garantismo", molte delle pratiche della giustizia penale brasiliana sono incompatibili con il modello di garanzie in essere e decisive per il processo di incarceramento di massa in corso, ponendo il Brasile tra $\mathrm{i}$ leader mondiali in questa questione.

La situazione è molto preoccupante, e l'eccesso di arresti provvisori rivela che i tribunali vedono la maggior parte degli imputati come presuntamente colpevoli, nasce da questa visione distorta numerosi altre pratiche operate in relazione al codice di procedura penale (nella parte in cui ci sono stati importanti modifiche apportate dalla riforma impronta prevalentemente garantista nel 2008, che per molti versi è stato semplicemente ignorato dai tribunali) e al quadro costituzionale previsto dalla Costituzione e dai trattati e le decisioni internazionali dei diritti umani che sono vincolanti per il Brasile. Anche con le nuove generazioni di studenti di giurisprudenza formate a contatto con il pensiero garantista, non entreabre una certa comprensione significativo raffreddamento delle pratiche antigarantiste del sistema della giustizia. 
Questa mancanza di cambiamenti significativi sottolinea, tra l'altro, l'effetto straniante ottenuto in lunghi processi di istituzionalizzazione che gli studenti principianti sono sottoposti a una carriera nel sistema di giustizia (che comprende corsi di formazione, gli standard di correzione, obiettivi di produttività, ecc), confermando in essi il modello generale di normalità imposto o, se applicabile, o, se del caso, adeguandoli a questo standard (di pratiche antigarantiste consolidate per la legge brasiliana). Questa percezione convergono spaventosamente comprensione di Albert Schweitzer, nel suo studio Decadimento e Rigenerazione della Cultura (Verfall und Wiederaufbau der Kultur), per avvertire che "un altro motivo per cui il rinnovamento della cultura è difficile è che, come realizatori del movimento, e in modo decisivo, solo le individualità possono essere prese in considerazione separatamente. La rigenerazione della cultura non ha nulla a che fare con i movimenti legati alla psicologia delle folle. (...) Dove le comunità hanno più influenza sull'individuo di quanto possa esercitare su di loro, inizia la decadenza (...) “. D’altra parte, nel prefazione di Diritto e Ragione: Teoria del garantismo penale, Norberto Bobbio fa notare che il più perfetto sistema del garantismo non riesce a trovare in se stesso la propria garanzia e richiede un intervento attivo da parte di individui e gruppi in difesa diritti che, anche dichiarati normativamente (positivi), non sono sempre protetti in modo efficace.

Domanda 25. Data questa prospettiva, cosa consiglierebbe nel campo dell'educazione giuridica in modo che noi, insegnanti di giurisprudenza, formiamo individui che sono veramente convinti della necessità di lottare per i diritti decorrenti del modello esistente di garanzie e nella misura in cui hanno una forza interna sufficiente per rifiutare qualsiasi tentativo di normalizzazione istituzionale delle pratiche antigarantiste?

L.F.: L'educazione giuridica è oggi, principalmente, educazione ai valori costituzionali, cioè ai valori della libertà, dell'uguaglianza, della pace e della democrazia. L'educazione civile a questi valori e alle loro garanzie e lo sviluppo dell'impegno politico e intellettuale, soprattutto dei giovani, saranno tanto più efficaci quanto più riusciranno a promuovere l'indignazione contro l'arbitro e il sopruso: l'indignazione, per esempio, contro la presunzione di colpevolezza che, come lei dice, sembra animare i tribunali brasiliani, e contro il conseguente abuso della custodia cautelare e degli arresti provvisori, che sono altrettanti sintomi della scarsa cultura istituzionale dei nostri operatori giuridici. Giustamente lei richiama quanto ha scritto Norberto Bobbio sull'intervento attivo delle persone in difesa dei diritti. Solo la lotta quotidiana e radicale in difesa dei diritti può garantirne l'effettività. In assenza di questa lotta i diritti sono destinati a sparire dall'orizzonte della politica e ancor prima degli stessi cittadini che ne sono i titolari.

Nell'insegnamento occorrerebbe inoltre mostrare che i valori costituzionali non sono soltanto valori etici e politici. Sono, prima ancora, valori di ragione. Dalla loro lesione possono provenire catastrofi in grado di distruggere non soltanto le nostre democrazie, ma anche le no- 
stre condizioni di vita e di sopravvivenza. E' questo un pericolo nuovo nella storia dell'umanità: di fronte alle catastrofi ambientali o a quelle nucleari, se non saranno introdotti limiti e controlli in breve garanzie - in grado di por fine all'attuale sviluppo industriale ecologicamente insostenibile e di bandire come illeciti gli assurdi armamenti nucleari, potremmo non arrivare in tempo a formulare i "mai più" che a metà del secolo scorso furono opposti agli orrori delle guerre e dei totalitarismi. La lotta per i diritti e per le loro garanzie è insomma un imperativo di ragione ancor prima che di giustizia. E' tutt'uno con l'impegno razionale, e non solo etico-politico, in difesa della democrazia, della pace e dell'abitabilità del pianeta.

Fine 